\title{
Jeux et enjeux d'une mobilité transfrontalière à contre-courant à la frontière Mexique-États-Unis
}

Counter-current cross-border mobility on the Mexico-United States border: stakes and issues

Marie-Carmen Macias

\section{OpenEdition}

\section{Journals}

Édition électronique

URL : http://journals.openedition.org/transcontinentales/796

DOI : 10.4000/transcontinentales.796

ISBN : 978-2-7351-1557-0

ISSN : 1775-397X

Éditeur

Editions de la maison des sciences de l'homme

Édition imprimée

Date de publication : 31 décembre 2010

ISSN : 1950-1684

Référence électronique

Marie-Carmen Macias, « Jeux et enjeux d'une mobilité transfrontalière à contre-courant à la frontière Mexique-États-Unis », Transcontinentales [En ligne], 8/9 | 2010, document 10, mis en ligne le 31

décembre 2010, consulté le 08 septembre 2020. URL : http://journals.openedition.org/ transcontinentales/796 ; DOI : https://doi.org/10.4000/transcontinentales.796

Ce document a été généré automatiquement le 8 septembre 2020

Tous droits réservés 


\section{Jeux et enjeux d'une mobilité transfrontalière à contre-courant à la frontière Mexique-États-Unis}

Counter-current cross-border mobility on the Mexico-United States border: stakes and issues

Marie-Carmen Macias

1 En situation d'asymétrie entre deux pays, les facteurs économiques tendent à être surévalués dans l'analyse de la mobilité internationale de la main-d'œuvre quelle que soit l'échelle des flux. La frontière entre le Mexique et les États-Unis constitue un cas unique de contact présentant un grand degré d'asymétrie entre un pays industrialisé et un pays encore en développement - quoique le Mexique figure parmi les pays d'émergents. Les flux de toutes natures entre les doublets urbains situés le long de la frontière, longue de $3141 \mathrm{~km}$, s'inscrivent dans la dynamique nord-sud, mais pas exclusivement. Nous présentons ici les premiers résultats d'une recherche en cours portant sur les mobilités résidentielles et pendulaires des frontaliers mexicains ayant décidé de déménager avec leur famille, du sud vers le nord de la frontière, de Tijuana (Basse Californie) vers la ville états-unienne de San Diego (Californie), tout en maintenant leur activité professionnelle dans la ville mexicaine. Ces travailleurs frontaliers (cross-border commuters en anglais ou transmigrantes en espagnol) réalisent des migrations pendulaires domicile-travail à contre-courant du flux principal et de la logique économique observée. Selon cette dernière, les frontaliers mexicains passent chaque jour aux États-Unis pour travailler, soit de façon légale (parce qu'ils détiennent une green card ou parce qu'ils possèdent la double nationalité) soit de façon illégale, afin de percevoir un salaire supérieur à celui qu'ils percevraient au Mexique et payé en dollar, devise plus stable que le peso.

2 Nous souhaitons discuter le concept de capital spatial dans le contexte d'un espace frontalier. Le poste-frontière de ce doublet urbain transfrontalier d'environ 3 millions d'habitants enregistre chaque année plus de 50 millions de passagers, ce qui en fait le port terrestre le plus transité au monde. L'objectif est de mettre en évidence les critères 
de décision qui président au choix du lieu de résidence en fonction de cette situation d'asymétrie frontalière ${ }^{1}$ et les moyens mobilisés, tant en termes de capital économique qu'en termes de capital social, pour rendre une telle décision opérationnelle.

\section{Dynamiques sociospatiales de la frontière et inégalités entre les frontaliers}

\section{La frontière comme ligne de ségrégation sociospatiale}

3 La mobilité résidentielle des frontaliers mexicains vers la ville américaine est un phénomène complexe à partir duquel on observe des logiques diverses. Outre un marqueur de la modernité, la mobilité croissante des acteurs socioéconomiques sert de moyen de différenciation sociale puisque l'espace paraît relativement plus fluide pour les classes sociales aisées.

4 La frontière joue un rôle de ségrégation sociospatiale pour les individus installés d'un même côté. On peut les diviser en deux catégories :

5 - les frontaliers, c'est-à-dire les individus ou les familles dont la vie est fortement liée à la situation frontalière bien qu'ils n'aient pas accès à l'espace transfrontalier ;

6 - les transfrontaliers, dont "l'espace vécu» se superpose - et se structure aussi fortement - à la ligne de discontinuité territoriale par le travail, la scolarité, les relations familiales, etc.

7 Entre le Mexique et les États-Unis, on constate qu'une portion de la population des villes frontalières ne franchit jamais la frontière. La première raison à ce phénomène relève d'un désintérêt pour ce qui se passe de l'autre côté et la seconde s'explique par une impossibilité légale à franchir la línea (la ligne ou la border). L'indifférence vis-àvis du voisin se manifeste plus souvent aux États-Unis, où les résidents peuvent théoriquement circuler librement de part et d'autre de la frontière. Cependant, les statistiques montrent qu'ils sont moins nombreux à le faire que les résidents des villes mexicaines qui, quant à eux, sont obligés d'obtenir un visa pour entrer aux États-Unis (figure 1). Dans les deux pays, les frontaliers ont une situation des plus précaires. Aux États-Unis, il s'agit de migrants mexicains illégaux qui évitent de rentrer au pays pour ne pas perdre toute chance de revenir vers les États-Unis. Le Mexique ne dispose pas de statistiques officielles à ce sujet mais, d'après les estimations de certains chercheurs locaux comme T. Alegría (2009)², près de la moitié de la population de Tijuana ne dispose d'aucun document lui permettant de passer légalement la frontière, car les familles tijuanenses les plus modestes se voient refuser leur visa par les autorités étatsuniennes.

8 La probabilité d'obtenir un visa d'entrée pour les États-Unis est en rapport avec le niveau des revenus et avec la situation professionnelle du candidat. Une étude du département d'économie de l'Universidad Autonoma de Baja California (Uabc) réalisée en Basse Californie en 2002 le confirme. Parmi les foyers dont au moins un des membres avait un visa, 51,5\% d'entre eux disposaient de revenus supérieurs à 5 salaires minimum; à l'autre extrémité, les familles recevant moins d'un salaire minimum ne représentaient que $2,1 \%$. À l'échelle individuelle, l'inégalité est encore plus manifeste: les personnes situées dans la strate supérieure des revenus représentent $74 \%$ de la population disposant d'un visa alors que celles percevant moins 
d'un salaire minimum ne représentent que $1,7 \%$ du total ; entre les eux, les individus gagnant jusqu'à 3 salaires minimum représentent $11,2 \%$ des habitants de Basse Californie possédant un visa, et ceux recevant entre 2 et 5 salaires minimums, 13,1\% (figure 1). Il s'agit là d'une première inégalité parmi la population de Tijuana qui concerne la possibilité de franchir la frontière.

Figure 1

Détention de visa d'entrée pour les États-Unis et niveaux de revenus en Basse Californie $\%$

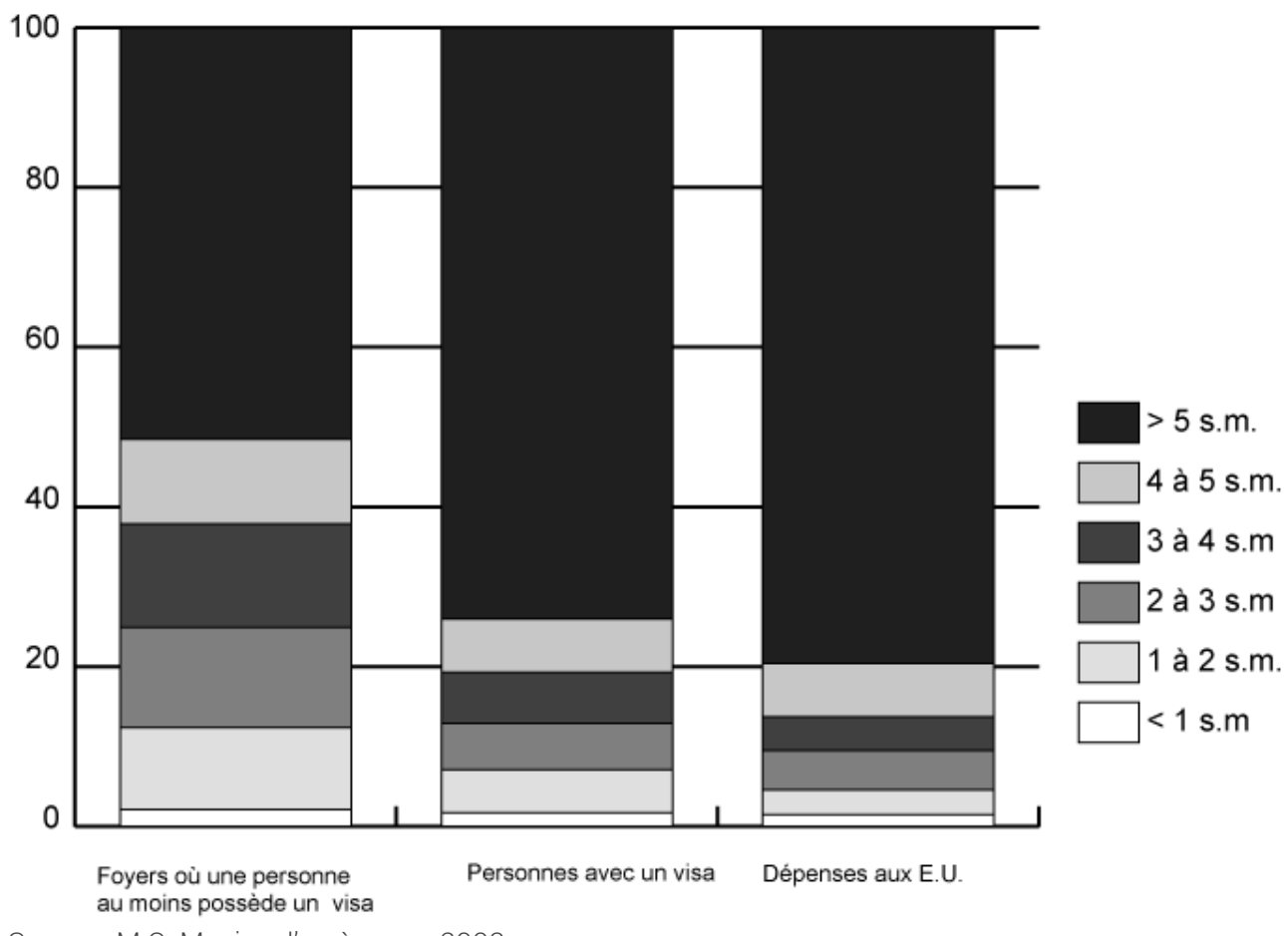

Source : M.C. Macias d'après UABC, 2003.

9 Cependant, la distinction entre frontaliers et transfontaliers ne rend que partiellement compte de la complexité des dynamiques car ces derniers constituent un groupe très hétérogène (Revel-Mouroz 1980). En effet, les mobilités transfrontalières sont motivées diversement. Les modalités du contact binational, ou plus précisément des passages transfrontaliers - échelles spatiotemporelles de l'interaction-, et la place qu'ils tiennent dans la vie des transfrontaliers est d'intensité variable comme le montre l'étude Who crosses the border? du San Diego Dialogue, réalisée en 1992 et illustrée dans la figure 2. Il n'en demeure pas moins que se constitue un système sociospatial transfrontalier fondé sur des dynamiques binationales de natures différentes (des travailleurs frontaliers, des consommateurs, du transport de marchandise, de la circulation des capitaux...), d'échelles spatiotemporelles diverses (locale, régionale, internationale, quotidienne à annuelle, etc.) et aux effets variés (transfrontaliers ou transnationaux $)^{3}$ selon que l'on se situe au nord ou au sud de la frontière.

Figure 2

\begin{tabular}{|l|l|l|}
\hline Motivations du passages transfrontalier $(>100 \%) *$ & Vers le Mexique & Vers les États-Unis \\
\hline
\end{tabular} 


\begin{tabular}{|l|c|c|}
\hline Affaires & $9,7 \%$ & $8 \%$ \\
\hline Travail & $6,0 \%$ & $30 \%$ \\
\hline Tourisme & $31,2 \%$ & $10 \%$ \\
\hline Loisirs & $43,4 \%$ & $19 \%$ \\
\hline Shopping & $31,4 \%$ & $62 \%$ \\
\hline Services & $11,7 \%$ & $5 \%$ \\
\hline
\end{tabular}

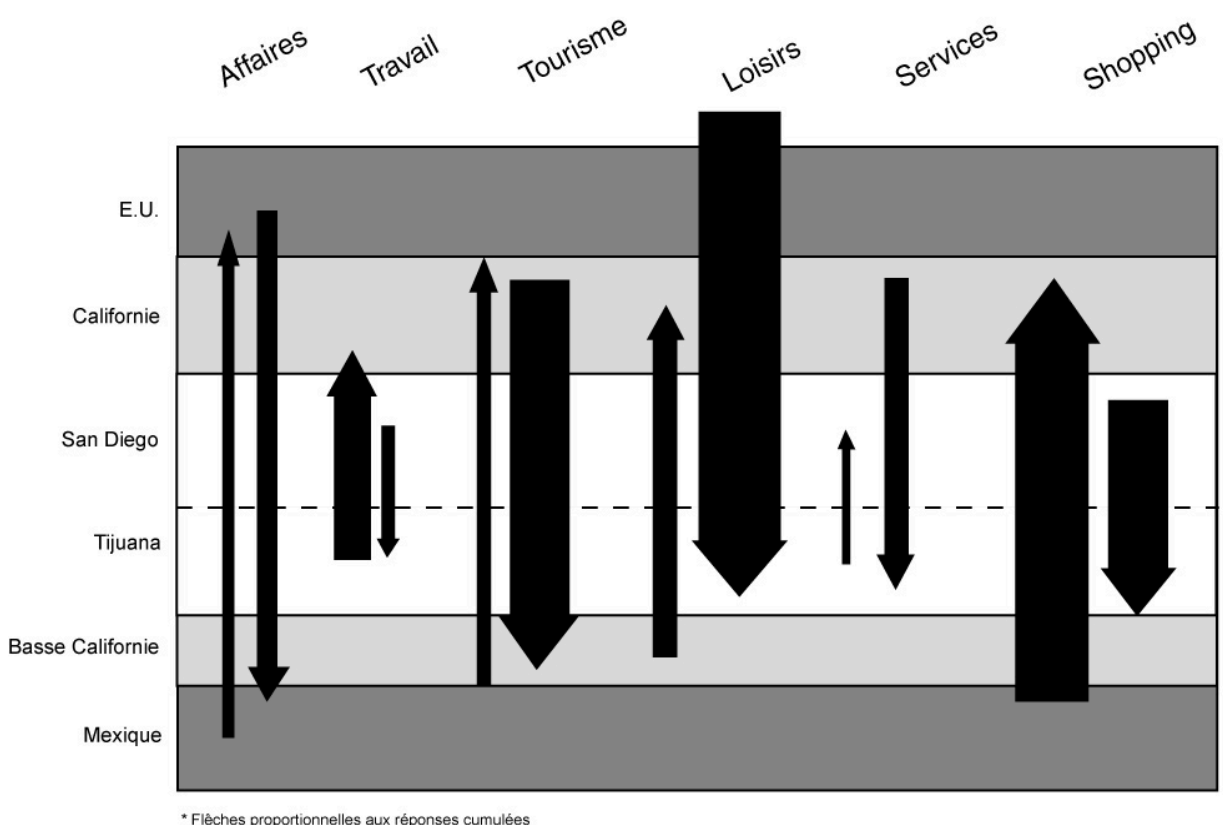

Motivations des passages transfrontaliers aux postes-frontières entre Tijuana et San Diego

Élaboré par M.C. Macias à partir de l'enquête de San diego Dialogue, Who Crosses the Border? 1994

\section{Les commuters à contre-courant : une catégorie de transfrontaliers longtemps invisibles?}

11 S'il est vrai que les membres de la classe aisée frontalière disposaient, depuis toujours, d'une double résidence ou d'une résidence secondaire au nord de la frontière, on observe, depuis les années 2000 , une mobilité résidentielle de frontaliers au profil social différent. Il s'agit d'un groupe plus large, aux revenus plus modestes, mais suffisamment solvable pour mettre en œuvre ce genre de projet familial. Mais, d'un point de vue conceptuel, il est difficile de les désigner autrement que par le terme de commuter transfrontalier compte tenu de l'hétérogénéité des situations.

12 Le changement de résidence ne se traduit pas à proprement parler par une émigration puisqu'au moins un membre de la famille, le plus souvent le mari, maintient une activité professionnelle au Mexique, ce qui le pousse à se déplacer de part et d'autre de 
la frontière, quoiqu'en sens inverse au flux principal des commuters. S'il est vrai que certains frontaliers entament des démarches pour acquérir un permis de résident - soit à travers le programme de visa pour investisseur ${ }^{4} \mathrm{E} 2$ soit à travers le processus de regroupement familial - la majorité des Mexicains vivant à San Diego sont arrivés avec un document légal pour y résider en tant que citoyens états-uniens ou résidents étrangers. En fait, leur installation aux États-Unis revient à adopter un mode de vie conforme aux définitions légales et conceptuelles de leur situation. Ainsi Julian ${ }^{5}$, un homme d'une soixantaine d'années, né aux États-Unis - donc de nationalité étatsunienne - dans une famille mexicano-états-unienne, a toujours vécu dans la ville mexicaine ; il y a été scolarisé dans les établissements publics puis il a occupé un poste de fonctionnaire même s'il ne possédait aucun document d'identité mexicain ${ }^{6}$, pas même le FM3 ou le FM2, permis de séjour pour étranger. D'un point de vue juridique, Julian a vécu clandestinement au Mexique jusqu'à la fin des années 1990, moment où l'État a promulgué la loi sur la double nationalité. Depuis une dizaine d'années, il vit aux États-Unis mais sa petite entreprise perdure au Mexique. Certains transfrontaliers nés aux États-Unis possèdent deux actes de naissance, un dans chaque pays, mais tel n'était pas le cas de Julian. Bien qu'illégale, cette pratique était très répandue parmi la population frontalière et, semble-t-il, parmi les migrants mexicains, avant la promulgation de la loi fédérale mexicaine sur la double nationalité. Enfin, certains individus ne possèdent qu'un passeport leur permettant de séjourner temporairement mais pas de résider légalement aux États-Unis même si, parfois, ils y possèdent un bien immobilier ou si un membre de la famille plus ou moins proche y jouit d'un statut de résident légal. Ces personnes ne sont pas comptabilisées parmi les commuters transfrontaliers.

Les travailleurs transfrontaliers résidant à Tijuana et travaillant dans le comté de San Diego sont plus nombreux que ceux qui effectuent les déplacements domicile-travail du nord vers le sud. Sur les $8 \%$ de la population active de la ville de Tijuana qui travaillaient aux États-Unis en 2001, jusqu'à $10 \%$ étaient des chefs de famille masculins ${ }^{7}$. Tant par leur nombre que par leur poids dans la population active totale de Tijuana, ces travailleurs jouent un rôle important dans l'économie locale. Cela explique les nombreuses études sur ce sujet (Acuña González 1988 ; Alegría 1990 ; Herzog 1990) alors que, sur le phénomène inverse, rien n'a encore été publié dans la littérature scientifique ${ }^{8}$.

Du nord vers le sud, 2532 personnes vivent dans le comté de San Diego et passent la frontière pour aller travailler au Mexique (recensement de population de 2000). Ce groupe ne représente que $0,2 \%$ de la population active résidant dans le comté de San Diego, soit 5,5\% du total des commuters (45 874) travaillant hors du comté.

Ces chiffres suggèrent qu'il s'agit d'un phénomène marginal mais il y a tout lieu de croire qu'ils sont sous-évalués. En effet, tous les commuters ne sont pas enregistrés comme résidents aux États-Unis - c'est le cas par exemple de ceux qui y possèdent une propriété sans titre de séjour. Par ailleurs, ce phénomène tend à prendre chaque jour plus d'importance dans le contexte de violence des villes de la frontière nord du Mexique $^{9}$, notamment depuis qu'en 2006 Felipe Calderon, le président de la République mexicaine, a lancé sa "guerre contre le narcotrafic » dont l'une des conséquences a été la fuite de certaines élites, parmi lesquelles des fonctionnaires publics voire les présidents municipaux des principales villes frontalières. 

différents processus de l'interaction binationale. Parmi ces commuters, les ingénieurs et les techniciens de l'industrie maquiladora ${ }^{10}$ qui exécutent l'essentiel de leurs tâches professionnelles au Mexique, sont payés par des entreprises transnationales ayant délocalisé une unité de production dont la gestion technique et administrative est assurée en partie par du personnel d'encadrement non mexicain. Le découplage entre le lieu de travail, l'origine géographique du revenu et le lieu de résidence prend part ici à la construction de territorialités transfrontalières fortement liées aux processus transnationaux de la globalisation. Ainsi, parmi les Ccc rencontrés, Paul travaille sous contrat aux États-Unis (selon la législation du travail de ce pays) en tant que gérant d'une usine maquiladora d'une multinationale et ne passe au Mexique que pour y effectuer certaines tâches de gestion.

àlement à ces flux de techniciens et d'ingénieurs de l'industrie maquiladora, depuis les années 2000 , les membres de la classe moyenne sont de plus en plus nombreux parmi les ccc. Cette catégorie se compose principalement de chercheurs et d'enseignants du supérieur travaillant dans des institutions publiques fédérales ou de l'État fédéré, de fonctionnaires locaux (tels que les présidents municipaux), de chefs d'entreprise et de membres des professions libérales notamment dans le secteur de la santé (médecins, dentistes, etc.), qui se sont installés dans le comté de San Diego ces dernières années tout en maintenant leurs activités professionnelles ou leurs affaires à Tijuana.

Selon le type d'activité, la fréquence des déplacements pendulaires varie. Ainsi, à l'instar de Julian, le chef d'une petite ou moyenne entreprise dans une branche de services comme la communication se rendra quotidiennement sur son lieu de travail, car la part du relationnel est très importante pour mener à bien son activité. Au contraire, Veronica, dentiste libérale, est mariée depuis dix-sept ans à un citoyen mexicano-américain qui a longtemps vécu à Tijuana tout en travaillant à San Diego. Depuis que la famille a déménagé à National City (États-Unis), Veronica fait passer l'éducation de ses enfants en priorité et n'exerce plus qu'une ou deux fois par semaine et sur rendez-vous à son cabinet de Tijuana. En outre, le contexte de violence induit des comportements spécifiques comme le fait d'éviter la routine dans les déplacements. Ainsi, par mesures de sécurité, Paul, notre gérant de maquiladora, ne visite son usine ni à heure ni à jour fixe, change le plus souvent d'itinéraire et ne communique ses heures de passage qu'à un seul de ses collaborateurs.

\section{Mobilité et capital spatial sous le prisme de la famille}

\section{Mobilité et capital spatial}

À travers les pratiques transfrontalières, se manifestent les inégalités socioéconomiques dans leurs dimensions sociotemporelles. La discontinuité spatiale est vécue différemment selon le statut social des frontaliers car, au-delà du déplacement dans un espace fluide plus ou moins ouvert, les possibilités d'accéder à des ressources variées sont plus importantes. L'examen des motivations montre que le degré d'asymétrie socioéconomique entre les deux Californie est un facteur important. Le passage de la frontière devient une ressource pour la population locale qui, selon les cas, peut ainsi disposer de revenus plus importants en travaillant aux États-Unis, 
épargner en profitant du différentiel des prix, protéger ses économies des dévaluations en ouvrant un compte dans une banque aux États-Unis, ou bien encore, améliorer l'éducation de ses enfants en les inscrivant dans un établissement scolaire du pays voisin, etc. Ces avantages liés à la mobilité constituent un capital sociospatial qui s'appuie sur des compétences spatiales.

Le concept économique de capital a été repris par J. S. Coleman (1988) afin d'intégrer la notion de choix rationnel des individus dans la construction d'une théorie sociologique. Depuis lors, le concept a été mobilisé dans des perspectives très différentes, notamment dans les travaux sur les réseaux sociaux de M. S. Granovetter (1973) et de ses disciples ou dans ceux de R. D. Putnam (1993). À la suite de P. Bourdieu (1979, 1980), qui ajoute au concept de capital social ceux de capital intellectuel, symbolique, etc. pour analyser et interpréter les relations de domination dans lesquelles le capital social contribue à la reproduction du pouvoir de certains groupes sociaux, quelques géographes commencent aussi à appliquer ce même concept à l'étude des dynamiques spatiales. Jacques Levy (1994:124) définit le concept de capital spatial comme l'« ensemble des ressources accumulées par un acteur lui permettant de tirer avantage, en fonction de sa stratégie, de l'usage de la dimension spatiale de la société ", et il inclut l'espace parmi les facteurs de différenciation sociale. Le concept de capital spatial tel que J. Levy l'utilise permet d'analyser les stratégies spatiales des acteurs dans les processus de la construction de territorialités.

21 La dimension des relations de domination n'entre pas directement dans notre étude, toutefois elle n'est pas absente des travaux des géographes, comme en témoigne le titre du dernier ouvrage de M. Lussault (2009): De la lutte des classes à la lutte des places. En effet, à travers le capital spatial, sont mobilisées des compétences telles que la maitrise des distances et des échelles, la capacité à aménager ou à découper l'espace, celle à franchir les limites, qui permettent la mise en place de stratégies d'occupation du territoire. Le concept de capital spatial, surtout utilisé pour analyser les stratégies résidentielles dans les espaces métropolitains, peut être de grande utilité dans le contexte des régions frontalières si nous tenons que la mobilisation de certaines compétences spatiales comme le maniement les distances et surtout celui du franchissement frontalier permet à certaines catégories de la population d'exploiter la discontinuité territoriale à des fins de reproduction sociale et familiale. Ainsi, en est-il des citadins qui acceptent de faire face aux dépenses supplémentaires qu'entrainent l'éloignement de leur résidence par rapport au centre-ville tout en préservant un niveau de services satisfaisant, ou encore de ceux qui acceptent le coût d'une résidence dans un quartier aisé pour avoir une offre éducative de meilleure qualité (Maurin 2004; Cally 2007).

Contrôler les distances permet d'utiliser ou d'intégrer les différences spatiales aux stratégies des acteurs dans un but spécifique, tel celui qui vise à assurer ou à garantir la reproduction sociale de la famille par l'instrumentalisation des différentiels socioéconomiques, ici, entre le Mexique et les États-Unis. Les espaces frontaliers sont ambivalents car la ligne internationale assure la double fonction d'obstacle et de contact ; ils sont d'une part des fronts, des lignes de confrontations, et d'autre part, des obstacles et des interfaces, c'est-à-dire des lieux de passage, d'interactions et de connexions (Foucher 1988). Selon les objectifs ou la position sociale des acteurs spatiaux, la discontinuité territoriale devient soit un interface soit une contrainte plus ou moins forte, voire une barrière dont il faut tenir compte pour la mise en œuvre 
d'une stratégie spatiale. Le caractère ambivalent de la frontière contribue à favoriser des processus de ségrégation sociospatiale que l'on surmonte grâce à son capital social et spatial.

\section{Une pratique spatiale au service d'une stratégie de reproduction familiale}

L'espace frontalier se caractérise par un certain degré d'incertitude dans la réalisation des activités, parmi lesquelles la libre circulation parait essentielle à la population locale en ce qu'elle permet l'accès à certains avantages, comme l'illustre l'analyse des motivations des passages transfrontaliers. Pendant longtemps l'incertitude restait liée à la conjoncture économique du Mexique. Les dévaluations monétaires depuis 1976, mais surtout depuis 1982, ont affecté aussi bien la population transfrontalière (en tant que consommateurs, main-d'œuvre, etc.) que la population frontalière stricto sensu (c'est-à-dire celle captive de l'offre des entreprises mexicaines), car le degré de dépendance économique de la frontière nord du Mexique vis-à-vis des États-Unis affecte aussi bien les usines délocalisées que les importations de biens d'équipement ou de consommation.

Le caractère incertain de la circulation transfrontalière n'a cessé de s'accentuer depuis les années 1990, depuis que les États-Unis ont décidé de manière unilatérale de sécuriser leur frontière sud en construisant un mur métallique pour mieux surveiller le narcotrafic et l'immigration clandestine. Cela a incité les transfrontaliers à changer leur mode de circulation voire à déplacer leur résidence vers la ville états-unienne. Emilio, mexicain originaire de Tijuana, marié à une citoyenne états-unienne anglosaxonne enseignante aux États-Unis et père de deux enfants, résidait dans un quartier de classe moyenne de Tijuana et, jusqu'en 2002, les enfants passaient chaque jour la frontière pour se rendre au collège. Le 11 septembre 2001, la frontière fut fermée subitement et les enfants n'ont pu rentrer chez eux. Au cours des mois qui ont suivi, le renforcement des contrôles a rallongé considérablement l'attente au poste-frontière (parfois jusqu'à deux ou trois heures). Pour épargner les enfants, le couple a alors décidé de déménager à Chula Vista, la ville la plus hispanique au sud de l'agglomération de San Diego, et Emilio effectue les déplacements pendulaires.

Le 11 septembre 2001 constitue un point de non retour dans ce mouvement de fermeture de la frontière (Macias 2008), à tel point que certaines familles mexicaines frontalières ont commencé à développer des stratégies pour préserver un espace de circulation fluide.

Étudiant la mobilité résidentielle des familles ouvrières de la ville frontalière mexicaine de Ciudad Juarez Zamorano-Villarreal (2003) utilise le terme d'itinéraire - trajectoire suggère l'idée de plan prédéfini pourtant absent - pour qualifier les adaptations des familles aux réalités socioéconomiques instables qui limitent l'action et empêchent l'exécution de stratégies à long terme. Le cas des classes moyennes frontalières est différent. En effet, ces mobilités relèvent du concept de stratégie de vie qui suggère une intentionnalité et la capacité de mettre en œuvre un projet. En revanche, le déplacement d'une résidence de Tijuana vers San Diego est conditionné à un certain niveau de solvabilité qui va de pair avec une connaissance fine du différentiel socioéconomique et juridique permettant d'exploiter la frontière comme une ressource (Constantin 1996) et la construction de réseaux socioéconomiques transfrontaliers. 
C'est pourquoi, ce changement de résidence peut être considéré comme un investissement dans la mesure où il suppose, pour la famille, un surcoût économique qui devrait apporter une rentabilité en terme social.

En effet, d'un point de vue économique, vivre à San Diego est désavantageux pour les ccc puisque la majorité de leur revenu provient du Mexique et est dépensé en dollars dans un pays à fort pouvoir d'achat. Évidemment, le coût d'une résidence à San Diego est plus élevé que celui d'une résidence à Tijuana. À ce coût, s'ajoute celui lié à l'incertitude du passage, surtout à l'allongement de la distance-temps - on estime le temps moyen d'attente entre 45 minutes et une heure voire plus, depuis le 11 septembre $^{11}$ - et à la possibilité d'une fermeture inopinée de la frontière. Ces dernières années, les transfrontaliers qui en ont les moyens adoptent le badge électronique SENTRI qui permet de passer rapidement, mais il s'agit, là encore, d'une démarche coûteuse ${ }^{12}$ tant en termes financiers qu'administratifs.

Sauf exception, les commuters à contre-courant qui ont participé à nos entretiens, en 2009, disposent non seulement de ressources confortables - ce sont de hauts fonctionnaires locaux, et surtout des membres de professions libérales (médecins, dentistes, avocats, etc.), des commerçants et des chefs d'entreprise - mais aussi de capital social en termes de réseaux transfrontaliers. Ils bénéficient de réseaux sociaux et familiaux importants dans le pays voisin puisqu'en principe le conjoint ou les fils peuvent résider légalement aux États-Unis ${ }^{13}$. Avant la loi de 1996, ces avantages étaient traditionnellement cultivés par la classe moyenne frontalière par la déclaration de la naissance d'un enfant dans chacune des deux villes jumelles de la frontière, autorisant la double nationalité mexicaine et états-unienne (Zavala de Cosio 1997).

De la même façon, cette mobilité transfrontalière résidentielle se fonde sur la connaissance fine d'un espace juridiquement et socioéconomiquement différencié qu'Alain Tarrius (1989) appelle le «savoir circulatoire ». Les compétences spatiales sont la base de la construction de territorialités transfrontalières qui reposent sur la séparation entre les espaces résidentiels et de travail. Cela correspond au découplage entre les logique productive et reproductrice de la famille. Jouer avec la frontière fait partie de la seconde.

30 La notion de stratégies familiales s'applique à des familles ayant une capacité de projection dans le futur qui dépasse les situations d'adaptation nécessaires à des événements conjoncturels ; ainsi, bien que le changement de résidence puisse être dû à des faits conjoncturels comme l'essor récent de la violence, il convient d'indiquer que, dans les entretiens réalisés, nombreuses sont les familles qui ont déménagé avant 2006, c'est-à-dire avant cette augmentation de la violence liée à la lutte contre les cartels de la drogue. Parmi les motivations de la mobilité résidentielle apparaissent d'autres facteurs, comme celui de travailler aux États-Unis non seulement pour les revenus mais aussi pour bénéficier à long terme d'une pension de retraite; d'obtenir la nationalité états-unienne pour bénéficier du Medicare (assurance sociale pour les personnes de plus de 65 ans) ; d'inscrire ses enfants dans le système éducatif de meilleure qualité donnant aussi accès au système de bourses universitaires, etc. Les quelques exemples qui suivent illustrent parfaitement ceci.

31 Originaires de Mexico, Felipe et Marisa se sont installés à Tijuana au milieu des années 1980 pour travailler en tant que chercheurs dans le système universitaire mexicain. Il y a sept ans, une université californienne a proposé d'engager Marisa qui a accepté, séduite entre autres par la perspective de cotiser à un système de retraite 
meilleur qu'au Mexique. La famille a alors déménagé à San Diego pour se conformer aux obligations imposées par les lois d'immigration aux États-Unis, et Felipe passe régulièrement la frontière plusieurs fois par semaine pour se rendre à son travail. Le couple a choisi de scolariser ses deux enfants dans un lycée et un college (université) de San Diego en vue de leur faire intégrer le système universitaire états-unien (s'ils avaient choisi le système universitaire mexicain, leurs enfants auraient dû quitter la région pour les universités privées de Monterrey ou de Mexico). Ce choix représente un coût élevé en termes de déménagement et de droits d'inscription dans un établissement privé, mais leurs enfants ont pu entrer dans un système d'enseignement supérieur de primer mundo (de pays industrialisé) ce qui, d'après Felipe, représente un avantage sur le marché mondialisé du travail. Ces élèves de classe moyenne qui avaient été bien formés dans l'un des meilleurs établissements scolaires de Tijuana ont pu facilement s'adapter au système éducatif états-unien, ce qui leur a permis d'obtenir une bourse pour les universités prestigieuses de Los Angeles et de San Francisco. S'ils n'avaient pas été résidents en Californie, ils auraient payé des droits d'inscription plus élevés réservés aux étudiants étrangers.

Pour Jessy, transfrontalière de 66 ans ayant acquis la double nationalité états-unienne et mexicaine, déménager sa résidence de l'autre côté de la frontière présentait l'avantage d'accéder aux prestations de Medicare. Atteinte d'une maladie chronique, elle doit faire face à des frais médicaux élevés qui, en raison de ses modestes revenus, sont désormais couverts par Medicare. Jessy a l'intention de continuer à travailler au Mexique car, depuis la crise de 2008, les quelques vacations qu'elle assurait à l'université de San Diego ont été supprimées, mais elle redoute la fin de son activité professionnelle puisqu'elle n'a jamais cotisé au système de retraite aux États-Unis.

Le 11 septembre 2001 a été le premier événement qui a perturbé cette mobilité résidentielle vers les États-Unis. La militarisation de la frontière avait introduit, au cours des années 1990, un facteur d'incertitude quant à l'ouverture permanente de la frontière qui s'est accentué après 2001. Résider légalement aux États-Unis permet de continuer à circuler malgré la politique du Homeland security, d'en atténuer les effets.

\section{En conclusion}

Les pratiques de la mobilité différencient les espaces frontaliers à diverses échelles spatiotemporelles de l'interaction binationale. Cependant, à l'échelle de la borderland, analyser les pratiques spatiales de la population locale permet de mettre en évidence le caractère pluridimensionnel des espaces d'appropriation. En fonction du degré de perméabilité de la ligne internationale, de l'accessibilité au territoire adjacent, de leur propre mobilité, les habitants de la frontière construisent des territorialités différenciées qui se superposent ou non à la discontinuité territoriale.

La maîtrise de la distance et surtout celle du franchissement de la ligne internationale, compétences spatiales mobilisées à des fins de reproduction familiale, sont d'autant plus valorisées que l'on observe la fermeture progressive d'une frontière qui fut traditionnellement perméable. Dans ce contexte spatial de discontinuité territoriale, la mobilité transfrontalière devient un enjeu. Cette mobilité aboutit à une fragmentation sociale de la population frontalière par le jeu d'une ségrégation spatiale de l'espace à l'échelle du doublet urbain qui est beaucoup plus radicale que les quartiers fermés des grandes métropoles latino-américaines. Dans le contexte de violence que connaît le 
Mexique, et tout particulièrement les villes de la frontière nord, franchir la frontière présente un enjeu vital.

En outre, bien que les mobilités résidentielles et les déplacements pendulaires domicile-travail ne soient pas à proprement parler des migrations, cette circulation, parce qu'elle a lieu dans le contexte d'une frontière entre le Nord et le Sud qui tend à se fermer, soulève des questions quant à l'installation durable des élites dans un pays du nord et présente quelques similitudes avec le problème de la mobilité internationale des élites vers les pays développés sous la forme d'une « migration par porosité ».

\section{BIBLIOGRAPHIE}

ACUÑA GONZÁLEZ, B. 1988. « Transmigración legal en la frontera México-Estados Unidos », Revista Mexicana de Sociologia, vol. 50, $\mathrm{n}^{\circ} 4$ (octobre-décembre) : 277-322.

ALEGRíA, T. 1990. « Ciudad y transmigración en la frontera de México con Estados Unidos », Frontera Norte, $\mathrm{n}^{\circ}$ 4, vol. 4, juillet-décembre :7-38.

ALEGRÍA, T. 2009. Metrópolis transfronteriza: revisión de la hipótesis y evidencias de Tijuana, México y San Diego, Estados Unidos. Tijuan-Mexico, El Colegio de la Frontera Norte \& Miguel Angel Porrúa.

BOURDIEU, P. 1979. La distinction - critique sociale du jugement. Paris, Minuit.

BOURDIEU, P. 1980. « Le capital social », Actes de la Recherche en Sciences Sociales, n 31:2-3.

CALLY, L. 2007. « Capital spatial, stratégies résidentielles et processus d'individualisation », Annales de géographie, $\mathrm{n}^{\circ} 64: 169-187$.

COLEMAN, J. S. 1988. "Social capital in the creation of human capital ", American Journal of Sociology, 94 (supplément Organizations and Institutions : Sociological and Economic Approaches to the Analysis of Social Structure) : 95-S120.

CONSTANTIN, F. 1996. «L'informel internationalisé ou la subversion de la territorialité », Cultures \& Conflits, $\mathrm{n}^{\circ} 21-22$ : 311-345, en ligne http://conflits.revues.org/index245.html. Consulté le 14 janvier 2011.

FOUCHER, M. 1988. Fronts et frontières. Un tour du monde géopolitique. Paris, Fayard, 1988.

GUICHONNET, P. et C. RAFFESTIN. 1974. Géographie des frontières. Paris, Presses universitaires de France (Sup - Le Géographe).

GRANOVETTER, M.S. 1973. « The strength of weak ties ", American Journal of Sociology, 78, 6, mai : 1360-1380.

HERZOG, L. A. 1990. « Border commuter workers and transfrontier metropolitan structure along the United States-Mexico border ", Journal of Borderland Studies, vol. V, nº $2: 1-20$.

LÉVY, J. 1994. L'espace légitime, sur la dimension géographique de la fonction politique. Paris, Presse de la Fondation nationale des sciences politiques.

LUSSAULT, M. 2009. De la lutte des classes à la lutte des places. Paris, Grasset. 
MACIAS, M. C. 2003. Étude géographique des mutations du commerce de détail. Le cas de la frontière nord et de la ville de Tijuana : exception ou modèle précurseur? Thèse de doctorat, université de la Sorbonne nouvelle.

MACIAS, M. C. 2008. «Coopération à la frontière Mexique/États-Unis : l'intégration sous haute surveillance ", in F. Taglioni \& J.-M. THÉODAT, Coopération et intégration : perspectives panaméricaines. Paris, L'Harmattan (Géographie et Cultures) : 35-50.

MAURIN, E. 2004. Le ghetto français, enquête sur le séparatisme social. Paris, Le Seuil.

OJEDA GÓMEZ, M. 1981. « México y los Estados Unidos : ¿ Interdependencia o dependencia de México ? » in S. GONZÁLEZ, La Frontera del Norte : integración y desarrollo. Mexico, El Colegio de México : 125-140.

PUTNAM, R. D. 1993. « The prosperous community : social capital and public life », The American Prospect, vol. $4 \mathrm{n}^{\circ}$ 13, 21 mars : 35-42.

REVEL-MOUROZ, J. 1980. « Perméabilité de la frontière Mexique-États-Unis, la mobilité des consommateurs, des travailleurs et des entreprises ", Bulletin de l'Association des géographes français, $\mathrm{n}^{\circ} 470$ : 227-234.

SAN DIEGODIALOGUE. 1994. Who Crosses the Border? A view of the San Diego/Tijuana Metropolitan Region, Rapport, avril, en ligne : http://www.sandiegodialogue.org/pdfs/sdtjcros.pdf, consulté le 14 janvier 2011.

TARRIUS, A. 1989. Anthropologie du mouvement. Caen, Paradigme, 185 p.

ZAVALA DE Cosio, M.E. 1997. « Cambios demográficos y sociales en la frontera norte de México : famila y mercados de trabajo ", Documents d'Anàlisi Geogràfica, nº 30 : 93-120.

ZAMORANO-VILLARREAL, Cl. C. 2003. Naviguer dans le désert. Itinéraires résidentiels à la frontière MexiqueÉtats-Unis. Paris, éditions de l'IHEAL.

\section{NOTES}

1. La notion d'asymétrie développée par Guichonnet et Raffestin (1974) fait référence au degré de dépendance entre deux espaces au niveau du fonctionnement local. Cette dépendance d'un côté par rapport à l'autre détermine la dynamique de l'espace proprement frontalier. Certains auteurs mexicains parlent donc d'interdépendance de la région frontalière du nord du Mexique en s'appuyant sur l'analyse de l'asymétrie des relations. Dans une perspective sociopolitique, M. Ojeda Gómez (1981) réfute la notion d'interdépendance dans le cas des relations mexicano-étatsuniennes car elle supposerait une «subordination mutuelle " alors que les disparités entre les deux pays créent plutôt une dépendance mexicaine à l'égard de son voisin et partenaire. En s'appuyant sur les domaines du commerce international, des flux touristiques, du volume des transactions frontalières et du mouvement des IDE, il montre que la puissance états-unienne face à un pays en voie de développement tel que le Mexique exclut toute possibilité de réciprocité dans la dépendance. Tout au plus, accepte-t-il l'idée d'une interaction (c'est-à-dire d'une influence réciproque).

2. L'auteur a obtenu cet ordre de grandeur à partir d'une enquête menée sur un échantillon de 8000 personnes sélectionnées à l'échelle du quartier (la colonia), pour le compte du municipe de Tijuana en 2000 (Estudio de geografía social de Tijuana, 2000). Il confirme les données plus anciennes de la Encuesta Socio-económica de la Frontera. 
3. Toutefois, cette catégorisation entre transfrontalier et transnational est en quelque sorte une simplification de processus entremêlés: certains flux relèvent de dynamiques à la fois transfrontalières (à l'échelle locale ou régionale) et transnationales (plus globales liées aux processus internationaux et/ou à la mondialisation).

4. Le « Investor/Business visa » est un visa de résident délivré aux personnes qui investissent de 100000 dollars (pour une résidence temporaire renouvelable) jusqu'à 1 million de dollars (statut de résident permanent) dans une entreprise aux États-Unis.

5. Tous les prénoms des personnes interviewées ont été changés.

6. Pour inscrire un enfant dans le système éducatif mexicain, les parents doivent produire son acte de naissance ou leur attestation de résident. De la même façon, les postes de la fonction publique ne peuvent être occupés que par des nationaux.

7. Données de la Encuesta Nacional de Empleo Urbano de l'Instituto Nacional de Estadística y Geografía (INEGI).

8. Cependant, depuis deux ans, la presse mexicaine comme celle des États-Unis communiquent beaucoup sur le départ de la population mexicaine de la frontière (surtout de Ciudad Juarez) vers les villes des États-Unis en raison de la "guerre contre le narcotrafic ». Mais, les chiffres cités dans certains articles ne sont pas étayés par des enquêtes solides. En outre, cette mobilité résidentielle est interprétée comme une migration définitive alors que, dans de nombreux cas, les personnes concernées maintiennent leur activité professionnelle au Mexique et se déplacent plus ou moins quotidiennement, selon l'activité exercée.

9. Depuis 2008, les journaux titrent sur «l'exode» des familles et des entrepreneurs de la frontière nord en citant parfois des chiffres dont on ignore la source.

10. Ce terme désigne une usine qui bénéficie d'une exonération des droits de douane pour pouvoir produire à un moindre coût des marchandises assemblées, transformées, réparées ou élaborées à partir de composants importés

11. San Diego Association of Governments California Department of Transportation, District 11, Economic Impacts of Wait Times at the San Diego-Baja California Border, Final Report, January 19, 2006, San Diego, $128 \mathrm{p}$.

12. La Secure Electronic Network for Travelers Rapid Inspection (SENTRI) correspond à un système d'identification rapide des frontaliers, obtenu auprès des services de migration, après des vérifications et un contrôle poussés des personnes ainsi que le paiement de frais d'environ 180 dollars par an. Les entrepreneurs frontaliers et le personnel d'encadrement de l'industrie maquiladora étaient les premiers détenteurs de ce passe magnétique conçu spécialement pour eux et mis en place avant 2001.

13. Précisons que les conditions de sécurité ne permettent pas d'entrer facilement en contact avec les informants; c'est pourquoi, nous ne présentons pas ici le cas de ceux qui passent la frontière légalement mais résident illégalement aux États-Unis.

\section{RÉSUMÉS}

L'étude du concept de capital spatial est faite à partir du cas de la mobilité résidentielle dans un espace transfrontalier. La recherche, encore en cours, porte sur les frontaliers mexicains qui ont décidé de déménager avec leur famille du sud de la frontière vers la ville états-unienne mais dont l'un des conjoints, la plupart du temps le mari, continue à avoir une activité professionnelle dans 
la ville mexicaine. Autrement dit, nous avons le cas de travailleurs frontaliers qui réalisent des migrations pendulaires domicile-travail à contre-courant de la logique économique bien connue qui voudrait que les frontaliers mexicains passent chaque jour aux États-Unis pour travailler, soit de façon légale - parce qu'ils détiennent une green card ou parce qu'ils possèdent la double nationalité - et percevoir un salaire plus élevé qu'ils pourraient espérer au Mexique et en dollar, devise plus stable que le peso. Les cas de figure étudiés mettent en évidence les motivations qui interviennent dans le choix du lieu de résidence au-delà d'une logique économique. En effet, l'asymétrie socio-économique leur est défavorable puisque ces personnes retirent l'essentiel de leurs sources de revenus d'une activité réalisée au Mexique mais ont fait le choix de dépenser leur argent aux États-Unis. D'autres facteurs interviennent donc dans leur choix effectué bien que celui-ci ne soit possible qu'à condition de disposer d'un certain capital économique mais aussi social. Ce faisant la maîtrise de la distance et surtout celle du franchissement de la ligne internationale constitue un avantage qui peut être instrumentalisé dans une logique de reproduction familiale d'autant plus valorisée à l'heure de la fermeture progressive d'une frontière qui fut traditionnellement perméable. Dans ce contexte spatial de discontinuité territoriale, la mobilité transfrontalière devient un enjeu dans la stratégie familiale.

This article studies the concept of spatial capital by looking at residential mobility in crossborder areas. Research, which is still being carried out, focuses on Mexican border residents that have decided to move to United States cities with their families, while one of the spouses (usually the husband) continues to work in Mexico. In other words, these frontier workers, who take part in pendular migration between home and work, travel in the opposite direction than that dictated by economic reasoning. Generally, Mexican border residents cross into the United States every day to work legally (because they have a green card or double nationality) or illegally, and to earn a higher salary that is paid in dollars (more stable than the peso). In the above case, however, migrants look beyond economic reasoning and take into account other factors when choosing their place of residence. The socio-economic asymmetry is unfavourable to these individuals, because they earn most of their money in Mexico, but choose to spend it in the United States. Therefore, other factors influence their choices, even though these choices are not possible without some economic and social capital. The ability to master the distance and international border crossings, for instance, is an advantage in family reproductive strategies. This is especially true given that the border, which used to be permeable, is gradually being closed. In this spatial context of territorial discontinuity, cross-border mobility becomes a key aspect of family strategy.

\section{INDEX}

Thèmes : migration, mobilité

Keywords : border workers, commuter, cross-border mobility, Mexico, Baja California, San Diego, Tijuana

Mots-clés : travailleurs frontaliers, commuter, mobilité transfrontalière

Index géographique : Mexique, Basse-Californie, San Diego, Tijuana

\section{AUTEUR}

\section{MARIE-CARMEN MACIAS}

Professeur agrégée en géographie, pensionnaire au Centre d'études mexicaines et centro-américaines (CEMCA-UMIFRE 16) à Mexico (2008-2009), http://www.cemca.org.mx 
mcmc@free.fr 